Supplemental information for

\title{
$\alpha$-Synuclein penetrates mucin hydrogels despite its mucoadhesive properties
}

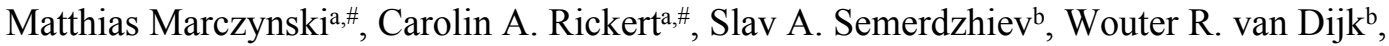 \\ Ine M.J. Segers-Nolten ${ }^{b}$, Mireille M.A.E. Claessens ${ }^{b}$, and Oliver Lielega,"
}

\footnotetext{
a Department of Mechanical Engineering and Munich School of Bioengineering, Technical University of Munich, Boltzmannstrasse 11, 85748, Garching, Germany

${ }^{\mathrm{b}}$ Nanobiophysics, Faculty of Science and Technology, University of Twente, Enschede, the Netherlands
}

\footnotetext{
*: correspondence: oliver.lieleg@tum.de

\#: equal contribution
} 


\section{Refined estimation of the polypeptide net charge of all $\alpha \mathrm{SN}$ variants used in this study}

In the main manuscript, the net charge of $\alpha \mathrm{SN}$ and the synthetic peptides was estimated by assuming full elementary charges per amino acid (if the pKa value of the respective amino acid was above/below pH 4). For a more detailed estimation, we here used two different protein calculators (public domain software): Protein Calculator v3.4 (http://protcalc.sourceforge.net/, 10/24/2019) and Prot pi (https://www.protpi.ch/Calculator/ProteinTool, 10/24/2019). For both estimations, the amino acid sequence of wildtype $\alpha$-synuclein was used ${ }^{1}$ :

MDVFMKGLSK AKEGVVAAAE KTKQGVAEAA GKTKEGVLYV GSKTKEGVVH GVATVAEKTK EQVTNVGGAV VTGVTAVAQK TVEGAGSIAA ATGFVKKDQL GKNEEGAPQE GILEDMPVDP DNEAYEMPSE EGYQDYEPEA.

Depending on the algorithm used, different net charge values for the $\alpha \mathrm{SN}$ protein were obtained. Those values are summarized in the following table:

Table S1: Estimated net charge of the five aSN variants used in this study. Values were determined for a pH level of 4.0 using the protein calculators listed in the tables. The calculated net charges are in good agreement with the determined net charge when elementary charges are assigned to amino acid side chains that are positively (Arg, His, Lys) and negatively charged (Asp) at pH 4.0.

\begin{tabular}{|c|c|c|c|}
\hline $\boldsymbol{\alpha}$-synuclein variant & $\begin{array}{c}\text { net charge } \\
\text { assuming full } \\
\text { elementary charges }\end{array}$ & $\begin{array}{c}\text { according to Protein } \\
\text { Calculator v3.4 }\end{array}$ & according to Prot pi \\
\hline wild type $\boldsymbol{\alpha S N}$ & +10 & +9.3 & +8.9 \\
\hline $\boldsymbol{\alpha S N} 1-60$ & +12 & +10.1 & +10.2 \\
\hline $\boldsymbol{\alpha S N ~ 1 - 1 0 8}$ & +16 & +12.7 & +12.7 \\
\hline $\boldsymbol{\alpha S N ~ 6 0 - 1 4 0}$ & -2 & -0.7 & -1.2 \\
\hline $\boldsymbol{\alpha S N}$ A30P & +10 & +9.3 & +8.9 \\
\hline
\end{tabular}

With all three approaches, comparable results are obtained. However, we would like to emphasize that none of those calculator software applications take any possible tertiary or quaternary protein conformations into account (which might be negligible for unstructured proteins such as $\alpha \mathrm{SN}$ ), nor do they consider inductive effects (which might be very well relevant for a $\alpha \mathrm{SN}$ polypeptide).

${ }^{1}$ UniProtKB - accession number P37840 (10/24/2019) 


\section{Comparison of fluorescence peak positions and positions of visual changes in phase contrast images of Fig. 1}

The data shown in Fig.1a and $\mathrm{b}$ stem from two different measurements, in which the image acquisition times were different. For the first measurement, only fluorescence images were acquired to analyze the intensity profiles during the penetration of $\alpha \mathrm{SN}$ into the mucin hydrogel. During those experiments, changes within the hydrogel structure were observed in phase contrast microscopy but not sufficiently documented for all time steps. That is why another set of experiments was conducted with the goal to colocalize the intensity maxima and the internal interface observed in phase contrast images. As shown in the figure below, the fluorescence intensity maxima show the same time dependency for both measurements.

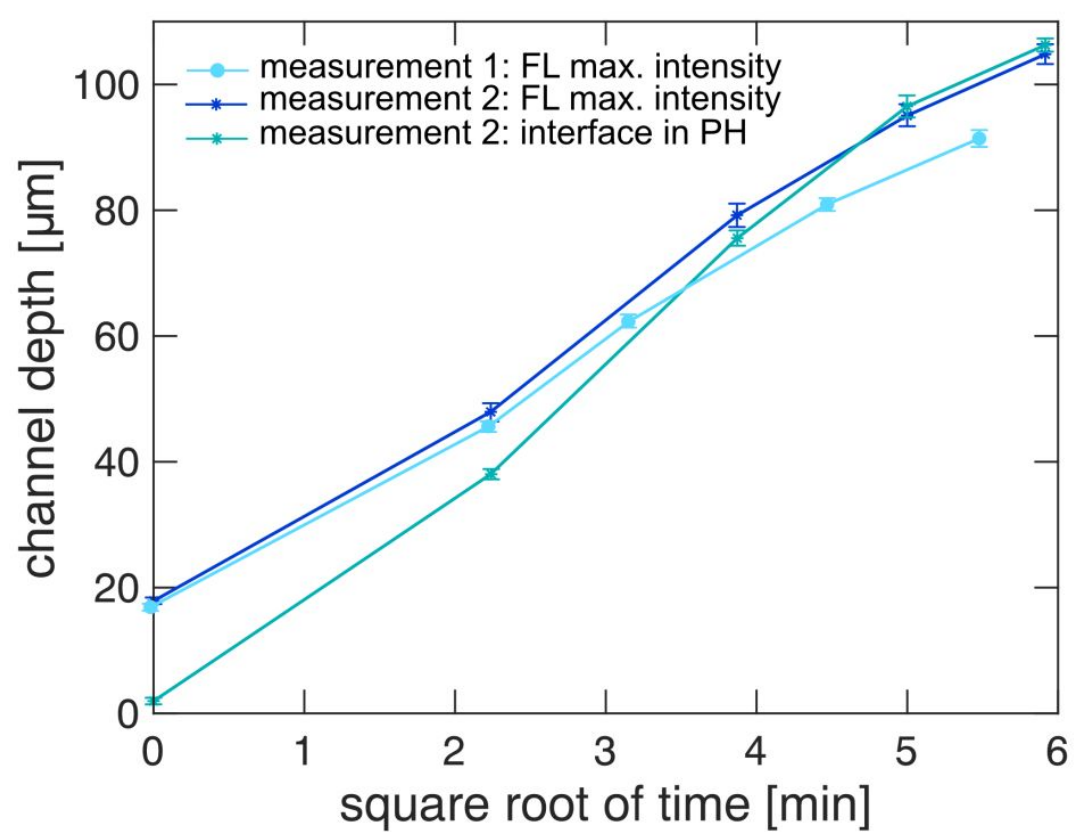

Figure S1: Positions of fluorescence intensity maxima and the internal gel interface visible in phase contrast images. The position of the fluorescence intensity maxima of the first measurement (bright blue line) and the second measurement (dark blue line) are plotted against the square root of time. The data from both measurements shows a time dependency of the peak position matching a root function. The positions of the internal gel interface that is visible in phase contrast images of measurement 2 is displayed in turquois. 


\section{MST measurements of wildtype $\alpha \mathrm{SN}$ and two mutant variants at different temperatures}

a) MUC5AC + aSN

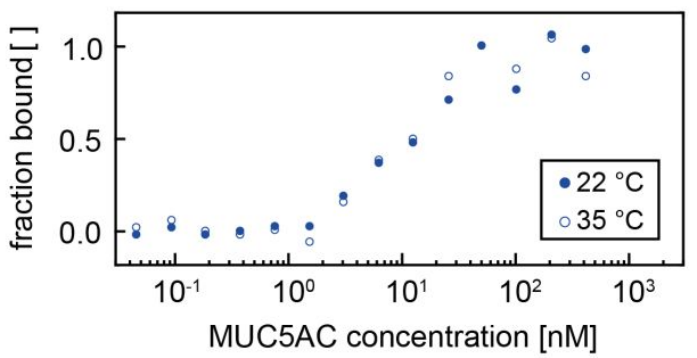

c) MUC5AC + aSN 1-108

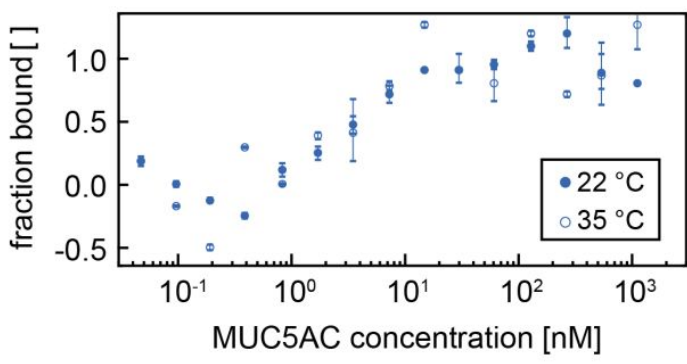

e) $\mathrm{MUC5AC}+\alpha \mathrm{SN} 1-108$
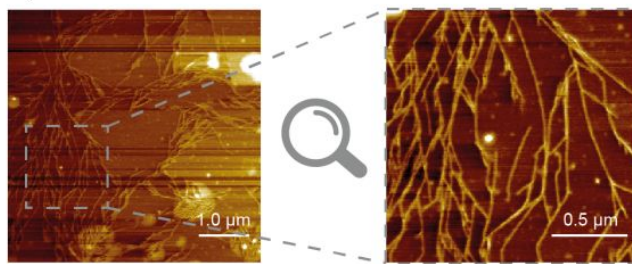

b) $\mathrm{MUC5AC}+\alpha \mathrm{SN} 60-140$

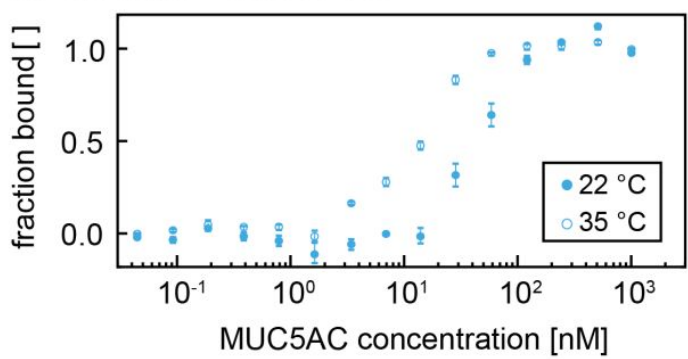

d)

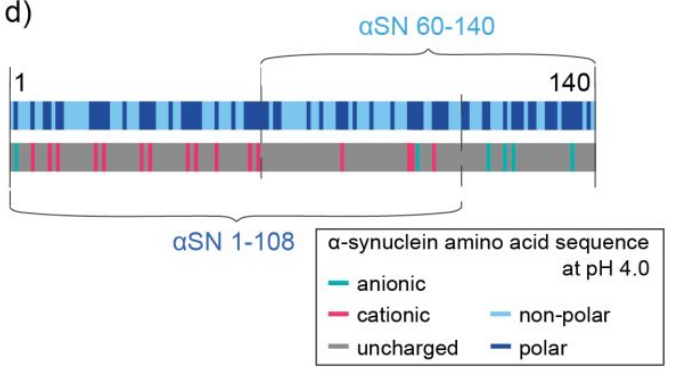

f) $M U C 5 A C+\alpha S N 60-140$
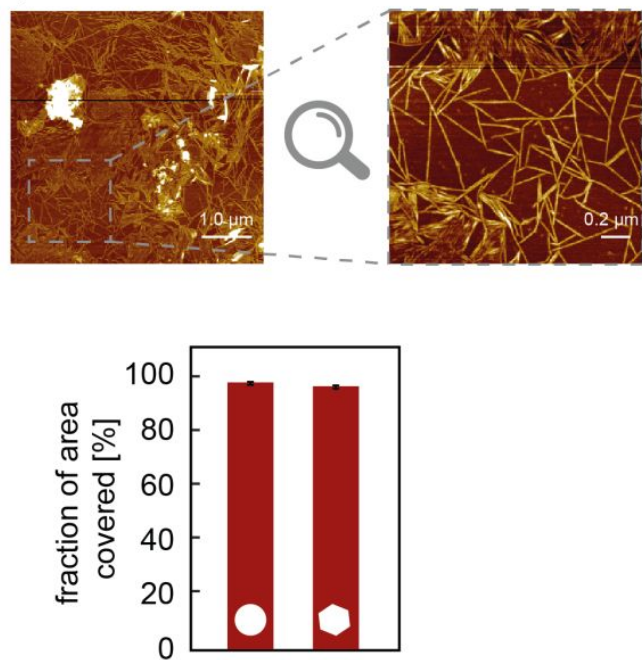

Figure S2: Mucin binding to different $\alpha S N$ variants as determined by MST and AFM. The binding affinity of wildtype $\alpha \mathrm{SN}$ and C-terminal truncated $\alpha \mathrm{SN} 1-108$ is independent of the ambient temperature (a, c). In contrast, the binding affinity of the $\mathrm{N}$-terminal truncated $\alpha \mathrm{SN}$ 60-140 exhibits a temperature dependency (b). The structure of the two $\alpha \mathrm{SN}$ is visualized in the charge and polarity maps shown for $\mathrm{pH} 4.0$ (d). AFM images of MUC5AC molecules incubated that have been incubated with $\alpha \mathrm{SN}$ 1-108 (e) and $\alpha \mathrm{SN}$ 60-140 (f), respectively, further demonstrate that both $\alpha \mathrm{SN}$ variants can bind to mucin molecules. $\alpha \mathrm{SN}$ 60-140 does not induce bundling of 
MUC5AC fibrils (g). The area fraction covered with fluorescent structures was determined and averaged for four frames $(n=4)$. Error bars denote the standard deviation.

MST measurements are conducted both at room temperature (i.e., $25{ }^{\circ} \mathrm{C}$ ) and at physiological body temperature (i.e., $37^{\circ} \mathrm{C}$ ). An altered binding affinity at an elevated temperature is only observed for $\alpha \mathrm{SN} 60$ 140. This $\alpha \mathrm{SN}$ mutant variant lacks the cationic $\mathrm{N}$-terminal domain and thus exhibits a negative net charge even at $\mathrm{pH} 4.0$; however, it comprises a large number of hydrophobic amino acids. The binding affinity of wildtype $\alpha \mathrm{SN}$ and $\alpha \mathrm{SN} 1-108$ is not affected by an increase in temperature. Since only hydrophobic interactions but not electrostatic interactions exhibit a temperature dependency, this indicates that the mucoadhesive properties of $\alpha \mathrm{SN}$ 60-140 might not be dominated by electrostatic interactions. In contrast, for the other two $\alpha \mathrm{SN}$ variants - which both carry a large number of cationic binding sites - binding interactions seem to be dominated by electrostatic forces. 
4. Phase contrast images of MUC5AC gels after penetration of different proteins and peptides

a)

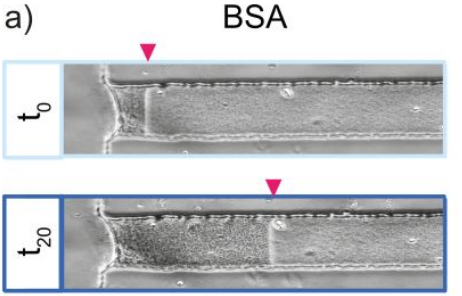

d)
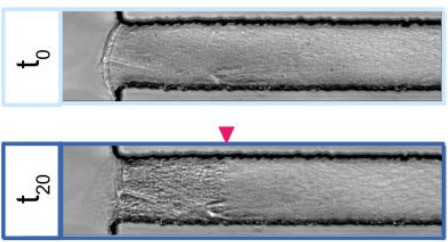

g)

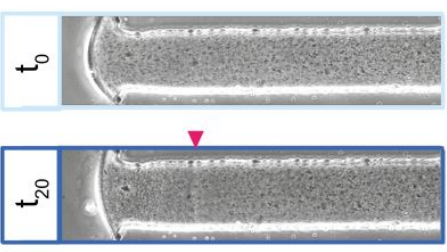

k) $\quad 5,6-$ TAMRA- $\left(Q_{5} K\right)_{4}$

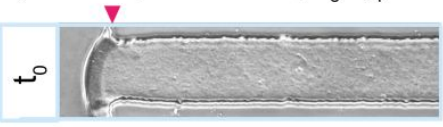

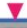

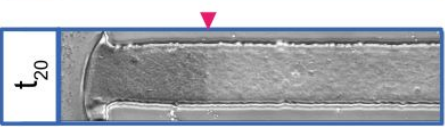

n)

$\mathrm{K}_{3} \mathrm{Q}_{18} \mathrm{~K}_{3}$
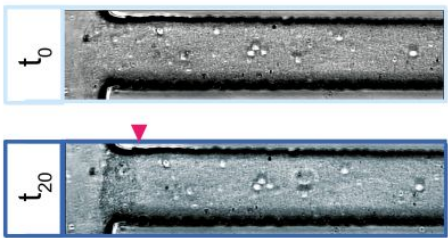

b)
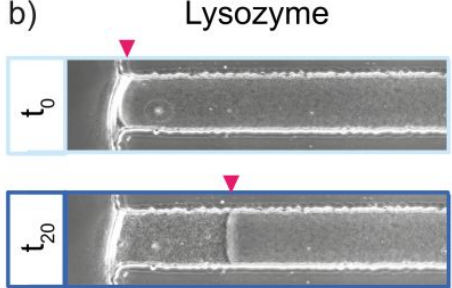

e)
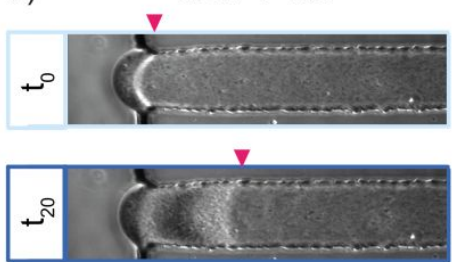

h) $\quad 5,6-T A M R A-(K K K)_{8}$

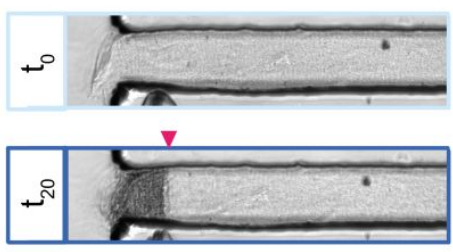

I) $\quad 5,6-T A M R A-\left(Q_{11} K\right)_{2}$
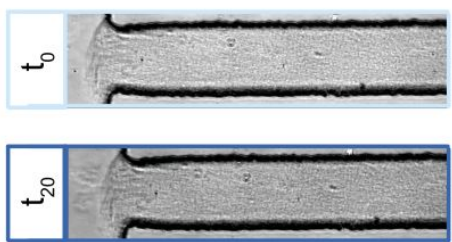

o)
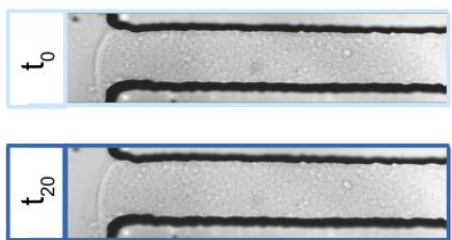

c) Poly-L-lysine

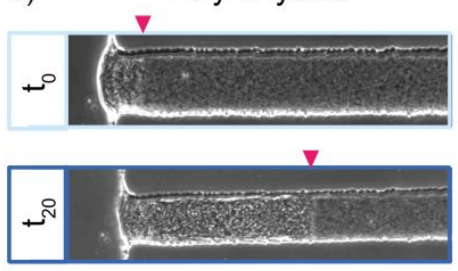

f) $\quad \alpha S N 60-140$
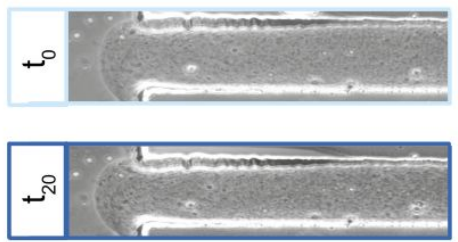

j) 5,6-TAMRA-(QQK)
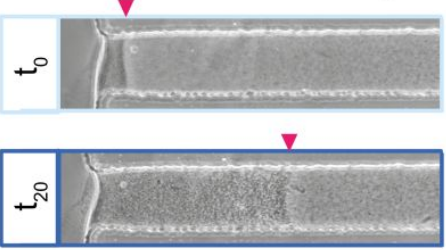

m) 5,6-TAMRA-(EEE) 8

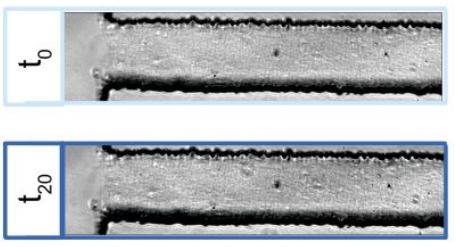

p) $\quad$ 5,6-TAMRA-Q ${ }_{21} \mathrm{~K}_{3}$
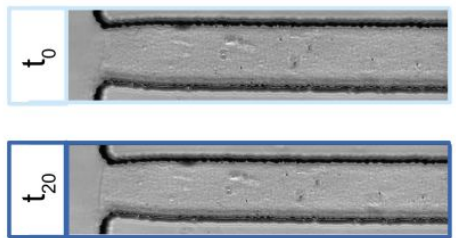

Figure S3: Phase contrast images of MUC5AC gels after injection of proteins/peptides ( $\left.t_{0}\right)$ and twenty minutes thereafter $\left(\boldsymbol{t}_{20}\right)$. The penetration of natural proteins $(\mathbf{a}-\mathbf{c}), \alpha \mathrm{SN}$ mutants $(\mathbf{d}-\mathbf{g})$, and synthetic peptides (h-p) was investigated. For each condition, at least three chips $(n \geq 3)$ were evaluated. 
Phase contrast images of the MUC5AC hydrogels were acquired after injection of the test molecules into the test reservoir. We refer to the time point of the acquisition of the first image as $t_{0}$. However, due to necessary adjustments of imaging settings after sample injection, the time between sample injection and acquisition of the first image differs between the different experiments, and we therefore sometimes detected an internal gel/gel interface even for images already at $t_{0}$.

\title{
5. $\alpha \mathrm{SN}$-induced rearrangement of MUC5AC gels at different conditions
}

\author{
phase contrast \\ fluorescence
}

a) $1 \%$ reduced MUC5AC $+100 \mu \mathrm{M}$ a-synuclein
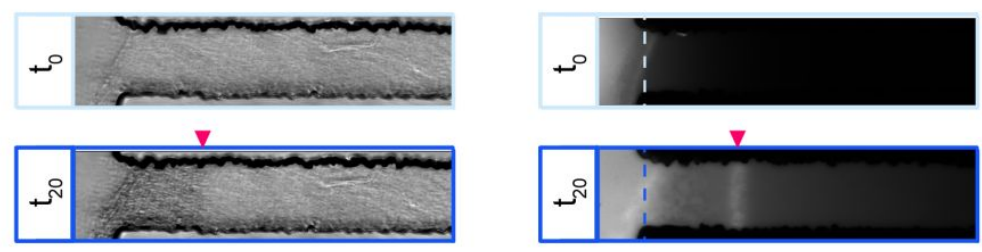

b) $3 \%$ MUC5AC $+100 \mu M$ a-synuclein
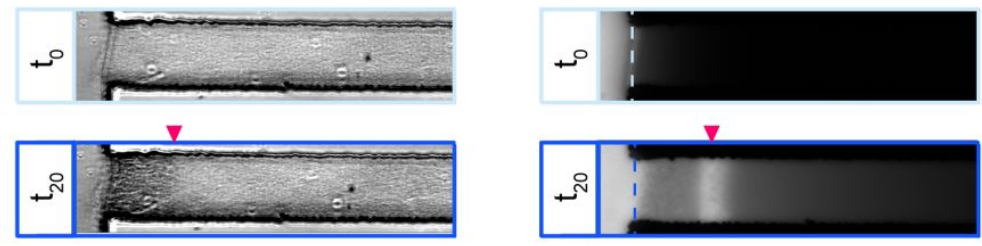

c) $1 \%$ MUC5AC $+10 \mu \mathrm{M} \alpha$-synuclein
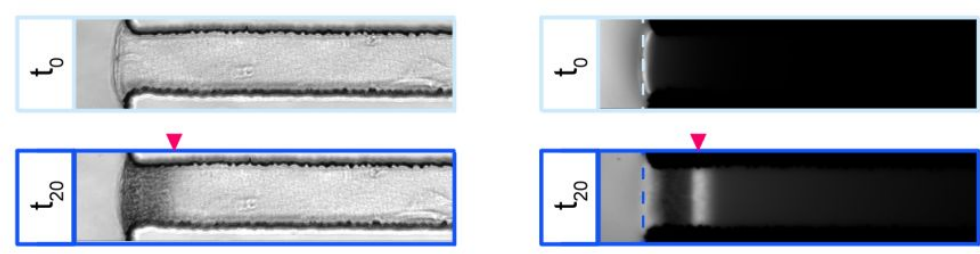

- - - - gel/buffer interface

Figure S4: $\alpha S N$-induced rearrangement of the MUC5AC gel microarchitecture at different conditions. a) For a 10 times lower concentration of $\alpha \mathrm{SN}(10 \mu \mathrm{M})$ than in the previous experiments $(100 \mu \mathrm{M})$ the rearrangement of the mucin gel structure can still be observed in phase contrast images. b) Even at a physiological mucin concentration of $3 \%(\mathrm{w} / \mathrm{v}), \alpha \mathrm{SN}$ rearranges the hydrogel network. c) Mucin hydrogels reconstituted in the presence of $1 \mathrm{mM}$ of the reducing agent DTT are also restructured by $\alpha \mathrm{SN}$. Arrows indicate the position of the internal gel/gel interfaces and dotted lines indicate the position of the gel/buffer interface in fluorescence images. For each condition, at least two chips $(n \geq 3)$ were evaluated. 